
allemande

47-1| 2015

L'Allemagne et l'Europe | La déprise de l'Empire napoléonien en Allemagne en 1813

\title{
Réformer la gouvernance de la zone euro : le dilemme allemand
}

Yann-Sven Rittelmeyer

\section{(2) OpenEdition \\ Journals}

Édition électronique

URL : https://journals.openedition.org/allemagne/451

DOI : 10.4000 /allemagne.451

ISSN : 2605-7913

Éditeur

Société d'études allemandes

Édition imprimée

Date de publication : 26 juin 2015

Pagination : 25-35

ISSN : 0035-0974

Référence électronique

Yann-Sven Rittelmeyer, « Réformer la gouvernance de la zone euro : le dilemme allemand », Revue d'Allemagne et des pays de langue allemande [En ligne], 47-1 | 2015, mis en ligne le 13 décembre 2017, consulté le 23 mai 2021. URL : http://journals.openedition.org/allemagne/451 ; DOI : https://doi.org/ 10.4000/allemagne.451 


\section{Réformer la gouvernance de la zone euro: le dilemme allemand}

- Yann-Sven Rittelmeyer*

\section{Introduction}

Alors que la fin de l'année 2013 a été marquée par une nouvelle réélection d'Angela Merkel à la tête de l'Allemagne, l'année 2014 a été riche en rendez-vous européens avec les élections européennes dans un premier temps, puis le renouvellement de l'ensemble des principaux postes européens (président du Conseil européen, président de la Commission européenne, président du Parlement européen et Haut représentant de l'Union pour les affaires étrangères et la politique de sécurité). À cela s'est ajoutée la mise en place de l'Union bancaire, une structure dont la création est étroitement liée aux problèmes mis en lumière par les crises de ces dernières années. Au-delà de son caractère technique, elle constitue une avancée importante en matière d'intégration supranationale.

Malgré cela, les crises financières et économiques qui touchent l'Europe depuis 2008 tardent toujours à être surmontées. Ces dernières ont en outre été doublées de crises sociales et morales dont les conséquences pourraient s'avérer encore plus durables. Aux crises déjà survenues dans la grande majorité des États membres et qui ont vu chuter ou partir de nombreux dirigeants en place (à l'exception notable de l'Allemagne) succède une crise profonde du projet européen. Latente depuis plusieurs années, elle s'est déjà manifestée lors des différents référendums qu'ont connus les ratifications de traités européens, ainsi qu'au travers des taux d'abstention aux élections européennes. Cette fois, non seulement l'abstention a été tout juste stabilisée et ce malgré les évolutions institutionnelles introduites par le traité de Lisbonne - lesquelles devaient pourtant contribuer à inverser la tendance -, mais surtout, les partis critiques à l'égard de l'intégration européenne ont connu leur plus large succès depuis la mise en place de ces élections. Cette expression populaire s'ajoute aux volontés de renationalisation

* Docteur en sciences politiques, chercheur associé au Centre d'étude de la vie politique (Cevipol) de l'Université libre de Bruxelles. 
de compétences européennes revendiquées par un nombre croissant d'États membres ou de préservation d'un statu quo défendu par d'autres par crainte d'une désintégration. Aujourd'hui, la structure européenne, basée sur le consensus et le compromis, continue d'absorber les ondes de choc qui la touchent. Les tendances nationalistes observées dans plusieurs États membres et la volonté exprimée au Royaume-Uni de remettre en question ses liens avec l'UE sont autant de pressions auxquelles doit faire face le projet européen. Le scrutin européen n'a donné lieu à aucun sursaut majeur au niveau des dirigeants européens. Les nominations aux principaux postes européens se sont faites quasiment comme à l'accoutumée (mis à part les modifications liées à l'entrée en vigueur du traité de Lisbonne). Et la nouvelle composition du Parlement européen devrait quasiment contraindre ce dernier à fonctionner sur le mode d'une «grande coalition » entre les deux principaux groupes.

Dans ce contexte, il peut paraître inadéquat de mener une réflexion sur les problématiques institutionnelles. En effet, les questionnements sur ces sujets ont cristallisé la défiance des citoyens au cours de ces dernières années. Pourtant, elle est devenue impérieuse non seulement pour des raisons légales et pratiques mais, plus profondément, parce que l'ensemble du projet est remis en question.

À force de voir l'Union européenne (UE) confrontée à ces crises et résultats négatifs à son égard, il est devenu légitime de se demander jusqu'à quand ce scénario peut durer. Peu à peu, un consensus s'est dégagé sur la nécessité de concentrer les efforts sur la zone euro. Mais quelle forme cette nouvelle gouvernance doit-elle et peut-elle prendre et quel rôle l'Allemagne va-t-elle jouer dans la détermination de cette dernière?

Afin de répondre à ces interrogations, il est dans un premier temps nécessaire d'évaluer le degré d'urgence de la situation institutionnelle et de la mettre en perspective avec les positions allemandes à ce sujet, dans la mesure où l'Allemagne joue un rôle déterminant quant à ces décisions. Enfin, nous analyserons les différentes propositions formulées jusqu'ici afin d'envisager les possibles perspectives pour la gouvernance de la zone euro.

\section{De la nécessité de (ré)former la gouvernance de la zone euro}

Les mesures prises dans l'urgence au cours des années de crise ont fait apparaître un ensemble qui n’a jamais été aussi complexe. Entre les membres de la zone euro qualifiés de «ins ", les « outs » et les «pre-ins ", les mécanismes et structures mis en place (traité sur la stabilité, la coordination et la gouvernance - TSCG -, « six pack », " two pack », sommets de la zone euro, Union bancaire, etc.), l'ensemble européen n'est plus seulement différencié, il est devenu désorganisé et insuffisamment légitimé démocratiquement. Plusieurs de ses équilibres fondateurs ont été mis à mal par la multiplication des structures parallèles. Face à ce manque de cohésion, le statu quo ne peut être une option sur le long terme. Dans une interview donnée au Handelsblatt à la fin de l'année 2012, l’ancien président de la Commission européenne, Jacques Delors, envisageait déjà - de manière peut-être un peu prématurée - la sortie de crise et déclarait: "Les pompiers peuvent se reposer, maintenant ce sont les architectes qui sont appelés ${ }^{(1)}$.

1 Interview de Jacques Delors par Thomas Hanke, « Mehr Integration in Europa? Dann los, Frau Merkel! », Handelsblatt, 28.12.2012. 
De manière similaire, en septembre 2013, celui qui fut pendant de nombreuses années l'un des plus influents architectes de l'ombre des négociations européennes, Pierre de Boissieu, écrivait dans un rapport remis au président français : «Il ne faut pas attendre les prochaines élections européennes pour agréer le cadre général d’une politique pour l'Europe. La fin de l'année 2013 et le début de l'année 2014 seront des moments cruciaux. Plus tard, il risque d'être trop tard ${ }^{(2)}$.

Les différentes institutions et mécanismes ont été adoptés sans qu’un débat ait lieu sur la cohérence de l'ensemble ainsi constitué et encore moins sur la finalité de ces mesures. Au fur et à mesure de ces décisions, une séparation entre les États membres de la zone euro et les États non-membres s'est opérée. Il a alors fallu répondre aux inquiétudes formulées par les États non-membres de la zone euro pour le moment mais qui ont l'obligation d'y entrer dans les prochaines années, lorsque leurs économies respecteront les critères établis. À ce titre, la nomination de l'ancien Premier ministre polonais Donald Tusk en tant que président du Conseil européen, mais également en tant que président des sommets de la zone euro, constitue une forme d'apaisement et il peut être en mesure d'ouvrir une voie pour la coordination entre les deux ensembles.

Le TSCG, signé le 2 mars 2012 par les États membres de l’UE moins le Royaume-Uni et la République tchèque, stipule que: «Dans un délai de cinq ans maximum à compter de la date d'entrée en vigueur du présent traité [...] les mesures nécessaires sont prises conformément au traité sur l'Union européenne et au traité sur le fonctionnement de l'Union européenne, afin d'intégrer le contenu du présent traité dans le cadre juridique de l'Union européenne. » Par conséquent, d'un point de vue juridique, les États membres de l'UE doivent parvenir à intégrer les institutions et obligations créées par ce traité au cadre juridique de l'UE avant le $1^{\text {er }}$ janvier 2018 dans la mesure où il est entré en vigueur le $1^{\text {er }}$ janvier 2013. Si ce délai peut encore sembler suffisant, la comparaison avec le temps qui fut nécessaire pour faire entrer en vigueur les précédents traités européens souligne l'urgence de la situation. En effet, le traité de Lisbonne a nécessité près de deux ans et demi, entre la déclaration de Berlin de mars 2007 et son entrée en vigueur. Or ce dernier n'était qu'une version légèrement modifiée des négociations menées dans le cadre de la Convention sur l'avenir de l'Europe dont les travaux avaient à eux seuls duré plus de deux ans, avant d'être signés mais sans être jamais ratifiés. Enfin, le traité de Nice entré en vigueur le $1^{\text {er }}$ février 2003 a été l'aboutissement d'un processus démarré lors du Conseil européen de Cologne en juin 1999 où fut décidé de convoquer une nouvelle conférence intergouvernementale. Compte tenu de la non-signature du pacte budgétaire par certains États membres de l'UE, son intégration au cadre juridique européen s'annonce particulièrement difficile.

Ces nombreuses évolutions posent également un problème de légitimation des décisions prises. Afin de pallier cela, l'idée avait été lancée de créer au Parlement européen une sous-commission dédiée à la zone euro au sein de la Commission pour les affaires économiques et monétaires. Mais cette idée a jusqu'à présent échoué en raison de divisions entre les présidents de groupe.

2 Rapport "Refaire l'Europe: esquisse d'une politique », par Pierre de Boissieu, Tom de Bruijn, Antonio Vitorino, Stephen Wall, septembre 2013, disponible en ligne: http://www.synopia.fr/?p=923. 
Les élections européennes ont rappelé avec force la nécessité de rétablir un lien entre les citoyens européens et le projet européen, et qu'il n'est de toute façon plus possible de supposer un consentement tacite. Par ailleurs, les pressions venues des gouvernements britannique et néerlandais pour revoir la répartition des compétences entre le niveau national et le niveau européen participent à l'ouverture du chantier. Largumentaire employé aussi bien en France qu'en Allemagne pour répondre à ces revendications ainsi qu'aux préoccupations des citoyens s'inscrit, dans une certaine mesure, dans cette optique.

La campagne électorale qui a précédé les élections de mai 2014 a en effet été marquée par une invocation régulière du principe de subsidiarité. Ce principe figurait en germe dans l'intégration européenne dès ses débuts, mais il a vraiment commencé à se développer à partir des années 90 et du traité de Maastricht. Destinée à délimiter l'intervention de l'UE aux champs définis par les traités et à ceux où son action est plus efficace qu'aux autres niveaux, la subsidiarité a toujours été un principe cher aux autorités allemandes car inspiré de la culture fédérale. Dans la campagne de 2014, ce principe se retrouvait aussi bien dans le manifeste du Parti populaire européen (PPE) que dans celui du Parti socialiste européen (PSE) ${ }^{(3)}$. Les discours des acteurs ont été marqués par l'omniprésence de ce principe, à l'instar de Martin Schulz déclarant que «Bruxelles ne doit pas s'occuper de tous les petits détails. Ce qui peut être réglé au niveau communal, régional ou national doit l'être à ces niveaux ${ }^{(4)}$. Ces paroles font écho à des formules régulièrement utilisées par Angela Merkel.

Si la subsidiarité est à l'heure actuelle la principale réponse apportée aux volontés de renationalisation qui sont exprimées, afin de mener à bien les réformes, l'approbation populaire sera requise. Or il n'existe aujourd'hui dans aucun des grands États européens de majorité claire pour faire progresser davantage l'intégration sur ce qui en constitue le cœur. En effet, en Allemagne $50 \%$ (vs $47 \%$ ) sont opposés à l'idée de transférer davantage de pouvoirs décisionnels à l'UE pour résoudre les problèmes économiques. Ce rapport est de $55 \%$ vs $45 \%$ en France et atteint $71 \%$ vs $27 \%$ en Grèce et $76 \%$ vs $19 \%$ au Royaume-Uni ${ }^{(5)}$. Seuls les Polonais se montrent légèrement favorables à cette perspective ( $44 \%$ pour, $41 \%$ contre). Si jusqu'à présent l'intégration a été perçue comme un phénomène fonctionnaliste continu ne pouvant faire l'objet de retour en arrière, cette perception semble donc être appelée à changer. Les recherches sur le concept de « désintégration » commencent d'ailleurs à se multiplier au sein de la communauté scientifique. Le fait que pour la première fois depuis la création de l'Union un budget pluriannuel en baisse par rapport au précédent ait été adopté pour la période 2014-2020 a constitué un symbole fort de la direction actuelle. En outre, la

3 «We do not want a centralized Europe that deals with every detail of people's lives ", " a EU that tackles the big issues together, leaving the smaller issues to the capable reponsibility of individual countries ", EPP Manifesto; "Les décisions doivent être prises au niveau le plus approprié (local, régional, national ou européen) », Manifeste du PSE.

4 «Brüssel darf sich nicht in jede Kleinigkeit einmischen. Was immer auf kommunaler, regionaler oder nationaler Ebene geregelt werden kann, sollte dort geschehen. » Interview commune de Martin Schulz et Jean-Claude Juncker, Der Spiegel, 12 (2014), p. 64.

5 "A Fragile Rebound for EU Image on Eve of European Parliament Elections ", Pew Research Center, mai 2014, p. 22. 
sociologie du vote du 25 mai 2014 ne laisse guère espérer d'amélioration: en France, le Front national a enregistré ses meilleurs scores chez les moins de 35 ans $^{(6)}$. Face à ces incertitudes et aux risques élevés que présente l'ouverture de la boîte de Pandore, nombre de dirigeants européens hésitent sur la marche à suivre.

Les crises ont donc révélé des faiblesses auxquelles il a été répondu en urgence, mais seule une réforme de plus vaste ampleur est en mesure de permettre à l'UE de retrouver un nouvel élan dans le temps d'après-crises. Avec l'Union bancaire, une porte a été ouverte. Dans le même temps, les nombreuses difficultés rencontrées dans l'établissement de cette dernière ont mis en lumière la complexité des négociations à venir. Face à la multitude des besoins qui rendent ce processus d'ensemble irrémédiable, la France et l'Allemagne, conscientes de leur rôle central en matière de réformes institutionnelles et désireuses d'influencer le processus, ont déjà formulé des propositions en ce sens. Peu à l'aise avec sa position de leader européen qu'elle doit assumer malgré elle ${ }^{(7)}$, l'Allemagne continue de privilégier les accords avec son partenaire d'outre-Rhin, mais les difficultés à lire les souhaits et objectifs de chacun rendent la tâche complexe.

\section{Interrogations et incertitudes sur la position de l'Allemagne}

La combinaison de sa réussite économique retrouvée et de la crise économique touchant les autres États membres a de facto placé l'Allemagne dans une position de leader. Dès lors, elle se trouve dans une situation où elle est considérée comme responsable des nouveaux développements et où son activisme a même été régulièrement sollicité. Le ministre des Affaires étrangères polonais s'était ainsi particulièrement fait remarquer en déclarant fin 2011 craindre " moins la puissance de l'Allemagne que son inactivité ${ }^{(8)}$. Mais l'Allemagne est également critiquée lorsqu'elle répond à ces appels et fait valoir ses conceptions. Ce paradoxe se retrouve d'ailleurs dans les opinions radicalement opposées suscitées par les Allemands et leur chancelière. D’un côté, l'Allemagne serait, dans le monde, le pays le plus positivement apprécié pour son influence ${ }^{(9)}$ et la chancelière serait la plus appréciée des dirigeants des grands États européens par les populations de ces derniers ${ }^{(10)}$. Mais d'un autre côté, les jugements positifs à l'égard de la politique de la chancelière sont particulièrement bas en Espagne et en Italie (39 \% et $36 \%$ ), et dramatiquement bas en Grèce $(10 \%)^{(11)}$. Ces opinions négatives se traduisent par des attaques verbales virulentes ou des caricatures liées au passé de l'Allemagne.

Ce rôle de leader désormais endossé par l'Allemagne a pour conséquence que l'Allemagne est d'autant plus observée et tenue pour responsable des problèmes dès lors qu'elle se montre hésitante ou récalcitrante. Sa volonté de préserver l'indépendance

6 "Élections européennes 2014. Comprendre le vote des Français », sondage IPSOS/Steria effectué par Internet via l'Access Panel d'Ipsos auprès de 2048 personnes, du 22 au 24 mai 2014.

7 Voir à ce sujet: Yann-Sven Rittelmeyer, «L'Allemagne, européenne malgré elle? », Études, décembre 2013, p. 583-592.

8 Discours de Radek Sikorski, « Poland and the Future of the EU », Berlin, 28.11.2011.

9 «BBC Poll: Germany most popular country in the world », 23.05.2013, http://www.bbc.co.uk/news/ world-europe-22624104.

10 Sondage BVA, « Popularités comparées en Europe des chefs d’État européens », mars 2012, http://www. bva.fr/data/sondage/sondage_fiche/1109/fichier_popularite_des_chefs_detat_en_europeb9320.pdf.

11 "The New Sick Man of Europe: the European Union », Pew Research Center, 13.05.2013. 
de la BCE lors des négociations sur la mise en place de l'Union bancaire a rapidement été interprétée comme un moyen de faire ralentir le dossier, voire une tentative de le faire échouer. Il en va de même pour les questions sécuritaires, où les attentes envers l'Allemagne sont fortes.

Cette position de leader est particulièrement périlleuse pour un État tel que l'Allemagne qui précisément, depuis la Seconde Guerre mondiale, s'est forgée une culture de retenue et de consensus. Les appels toujours plus nombreux et pressants pour que l'Allemagne assume un rôle plus important sur les théâtres de conflits reflètent bien les difficultés posées par ce statut. Les discours prononcés par le président de la République fédérale ou par la ministre de la Défense font certes écho à ces sollicitations, mais de pareilles évolutions ont besoin de temps pour aboutir. Après la réunification, l'Allemagne n'a guère posé la question de son rôle dans l'UE et sur la scène mondiale. Or ce tournant de l'histoire lui a permis d'accroître son territoire et sa population, tout comme la fin de la Guerre froide et l'éloignement du souvenir de la Seconde Guerre mondiale lui ont permis de retrouver un statut d'État «normal ». Les crises actuelles ont creusé de surcroît l'écart sur le plan économique. Compte tenu de ces éléments, l'Allemagne doit déterminer la bonne attitude à adopter vis-à-vis de ses partenaires européens et le rôle qu'elle entend exercer en Europe. De même, elle doit parvenir à trouver la juste limite entre la défense de ses intérêts et le renoncement à ces derniers au nom de l'intérêt européen.

Les déclarations de responsables politiques ou d'intellectuels allemands montrent que l'Allemagne doit toujours mener le débat introspectif qui lui permettra d'apporter une réponse à ces questions. À la veille des célébrations du $50^{\mathrm{e}}$ anniversaire du traité de l'Élysée et alors que la question de l'attitude de l'Allemagne par rapport à l'intervention française au Mali était au cœur de l'actualité, le ministre allemand des Finances, Wolfgang Schäuble, avait surpris en déclarant - à contre-courant de la tendance actuelle - que «l'Allemagne ne veut plus être une grande puissance en politique étrangère » en ajoutant « comment le pourrions-nous après Hitler et Auschwitz? L'histoire laisse longtemps des traces ${ }^{(12)}$. Cette même position, justifiant toujours l'attitude actuelle de l'Allemagne par rapport à son passé, est partagée par l'ancien chancelier Helmut Schmidt qui estime que les Allemands sont exclus du leadership en Europe pour les prochains siècles du fait de leurs responsabilités dans les deux conflits mondiaux ${ }^{(13)}$. Il répondit ainsi à une question sur son opinion quant à l'appel du magazine The Economist pour que l'Allemagne assume son rôle de leader. Dans un dossier spécial intitulé « Le colosse réticent " paru quelques jours auparavant, l'hebdomadaire britannique s'était aussi inscrit parmi ceux estimant nécessaire que l'Allemagne assume son statut de leader européen ${ }^{(14)}$. Selon l'argument avancé, la question n'est pas « est-ce que l'Allemagne peut conduire l'Europe vers un meilleur futur? ", mais « est-ce que l'Allemagne est prête à le faire? ».

Ainsi, la place qu'elle occupe aujourd'hui confère à l'Allemagne une responsabilité quant au devenir de l'Union. Le sociologue allemand Ulrich Beck, particulièrement critique à l'égard de la politique allemande actuelle, a contribué à alimenter le débat

12 Interview de Wolfgang Schäuble et d'Alain Minc, "Keine Angst vor der Macht Deutschlands », Handelsblatt, 21.01.2013.

13 Interview de Helmut Schmidt, " Der Euro ist prima », Handelsblatt, 17.06.2013.

Dossier spécial «Europe's reluctant hegemon », The Economist, 15.06.2013, p. 3-20. 
sur le rôle de l'Allemagne en Europe avec son ouvrage "L'Europe allemande " publié en $2012^{(15)}$. Il reconnaît la responsabilité de l'Allemagne et le problème de l'histoire, et pense que les Allemands veulent être des " professeurs » faisant valoir leur autorité morale et leur savoir, plutôt que des leaders ou dirigeants.

Au sein des intellectuels allemands ayant connu la guerre puis ayant vécu l'ensemble du processus de reconstruction de l'Allemagne et simultanément de construction de l'Europe, le fait que l'Allemagne défende désormais plus explicitement ses intérêts que par le passé suscite un certain malaise. En assumant ses conceptions et ses volontés, l'Allemagne les prescrit quasiment aujourd'hui de facto aux autres États membres en vertu de son poids économique - et donc politique. Jürgen Habermas a ainsi exprimé la crainte que l'Allemagne revienne "à nouveau à cette "position semihégémonique" "(16). L'ancien ministre des Affaires étrangères Joschka Fischer est allé plus loin encore, prévenant qu'il serait à la fois «tragique et ironique » si l'Allemagne provoquait pour la troisième fois la « destruction de l'ordre européen », cette fois par des " moyens pacifiques et avec pourtant de bonnes intentions " ${ }^{(17)}$. Cette problématique quant au rôle et à la place de l'Allemagne sera l'une des clés lors des négociations institutionnelles et un défi délicat pour les responsables politiques allemands.

La cristallisation autour de cette nouvelle « question allemande » a conduit Wolfgang Schäuble à faire évoluer sa position et à la préciser. Dans une tribune intitulée « Nous Allemands, nous ne voulons pas d'une Europe allemande ", il a reconnu que l'Allemagne a une responsabilité particulière dans la stratégie adoptée en commun pour résoudre la crise qui frappe la zone euro, avance qu'elle est prête à assumer ce leadership, mais seulement en coopération, en premier lieu avec la France ${ }^{(18)}$. Si cette position peut permettre de répondre à certaines des interrogations posées à l'Allemagne, encore faut-il qu'elle soit en mesure de trouver les voies du compromis avec la France.

\section{La voie franco-allemande}

$\mathrm{Au}$ cours de ces dernières années, à plusieurs reprises, l'ouverture d'un chantier institutionnel a été remise à plus tard. Les appels venus d'Allemagne ont bien souvent trouvé sur leur route les souhaits français de remettre la discussion à plus tard. L'accord finalement trouvé entre les deux partenaires a consisté à repousser le débat sur les questions institutionnelles après les élections européennes de 2014. Cela avait encore été confirmé par l'ancien Premier ministre français, Jean-Marc Ayrault, dans une interview donnée en mars 2014 à un quotidien allemand ${ }^{(19)}$. Du côté allemand, le

15 Ulrich BEck, Das deutsche Europa. Neue Machtlandschaften im Zeichen der Krise, Berlin, Suhrkamp, 2012.

16 Jürgen Habermas, " Repolitisons le débat européen », Le Monde, 25.02.2014.

17 «It would be both tragic and ironic if a restored Germany, by peaceful means and with the best of intentions, brought about the ruin of the European order a third time », Joschka Fischer, 25 mai 2012, http://www.project-syndicate.org/commentary/the-threat-of-german-amnesia\#vEGltoi6A6TR J64F.99.

18 Tribune de W. Schäuble, «We Germans don't want a German Europe », The Guardian, 19.07.2013, http://www.theguardian.com/commentisfree/2013/jul/19/we-germans-dont-want-german-europe.

19 Dans cette interview, Jean-Marc Ayrault avança : «Les discussions sur la gouvernance européenne vont démarrer sérieusement après les élections européennes de mai. Le couple franco-allemand jouera un 
plus actif à ce sujet est sans doute le ministre des Finances, Wolfgang Schäuble, qui a à maintes reprises demandé que les traités européens soient modifiés afin de finaliser l'Union bancaire et qui fin mars 2014 avançait également: "Le débat sur les modifications de traités sera à nouveau à l'ordre du jour après les élections européennes. Le gouvernement fédéral plaidera pour qu'au moins au niveau de la zone euro, on obtienne des améliorations institutionnelles " ${ }^{(20)}$. L'idée de modifier les traités s'est peu à peu éclipsée au profit de la recherche de solutions à traités constants ou via de nouveaux accords. En avril 2014, le président du Conseil européen avait lui aussi ouvertement déconseillé de chercher à modifier le traité de Lisbonne vu le contexte actuel et préconisa de prendre autant de mesures que possible à traités constants ${ }^{(21)}$. Témoin de la prudence qui s'est progressivement développée, le contrat de coalition, signé fin 2013, se contente de dire que les bases juridiques de l'Union économique et monétaire vont être " adaptées ».

Pourtant, en mai 2013, dans le cadre des travaux liés au 50e anniversaire du traité de l'Élysée, la France et l'Allemagne se sont employées à trouver les voies de convergence aussi bien pour ce qui concerne les politiques économiques à mener pour sortir de la crise que pour en dessiner les institutions adéquates. Après plusieurs semaines difficiles entre les deux États, deux textes ont été présentés, nés en grande partie grâce au mouvement insufflé par l'anniversaire du traité: le rapport sur la compétitivité industrielle européenne et les propositions communes " pour renforcer l'Europe de la stabilité et de la croissance » ${ }^{(22)}$. Ce second texte avait été annoncé le 22 janvier 2013 comme contribution à venir pour le Conseil européen de juin 2013. Si son titre, en évoquant à la fois la stabilité et la croissance, mettait particulièrement en évidence la nature de compromis du document, ce dernier contenait néanmoins quelques propositions concrètes nouvelles, notamment pour ce qui concerne la gouvernance de la zone euro. La France et l'Allemagne proposaient ainsi de renforcer la gouvernance de la zone euro à l'issue des élections européennes et en formulaient les grandes lignes: des sommets de la zone euro plus réguliers, un président de l'Eurogroupe à temps plein avec des moyens renforcés, ou encore un contrôle parlementaire de la gouvernance de la zone euro assuré par des structures spécifiques au sein du Parlement européen pour garantir un contrôle démocratique et une légitimité appropriés, ainsi qu'un contrôle démocratique assuré au niveau national pour des décisions relevant des compétences nationales. S’ils ne tranchaient par exemple pas sur la question de l'opportunité d'un budget spécifique pour la zone euro, ils ont cependant pris position sur le contrôle démocratique à opérer et sur la volonté de renforcer et d'autonomiser encore ces structures de la zone euro. Ces mesures marquaient encore un peu plus

rôle important à ce sujet » (traduction de « Die Diskussionen über die europäische Governance werden nach den Wahlen zum Europaparlament im Mai ernsthaft beginnen. Das deutsch-französische Paar wird da eine wichtige Rolle spielen »), in: "Jean-Marc Ayrault: 'Frankreich kann sich reformieren' ", Handelsblatt, 11 mars 2014.

20 Interview de Wolfgang Schäuble, «Russland hat mehr zu verlieren », Handelsblatt, 27 mars 2014 , p. 4.

21 Interview de Herman van Rompuy pour la Süddeutsche Zeitung, 19 avril 2014, p. 8.

22 Contribution franco-allemande " La France et l'Allemagne ensemble pour renforcer l'Europe de la stabilité et de la croissance », 30 mai 2013, http://www.elysee.fr/communiques-de-presse/article/ contribution-franco-allemande/. 
l'acceptation par l'Allemagne, opérée depuis 2010, du développement d'une " gouvernance économique " au sein de l'UE et donc d'une dissociation de plus en plus nette au sein de l'UE entre les États membres de la zone euro et les autres. Par cette évolution, la chancelière démontrait à la fois son pragmatisme et son aptitude au compromis, la France ayant depuis plusieurs années poussé au développement de telles structures selon des modèles laissant l'essentiel du contrôle aux États.

Ces impulsions franco-allemandes trouvent leur prolongement, au niveau européen, dans les différents rapports commandés au président du Conseil européen. Jusqu’à présent, ces derniers n’ont pas encore suscité une adhésion suffisante au sein des chefs d'État et de gouvernement pour être traduits en acte. La situation présente continue par conséquent d'être un terrain fertile pour des propositions multiples, notamment des deux côtés du Rhin.

\section{Une multitude de projets, confrontés à la question démocratique}

Les problèmes actuels auxquels est confrontée l'Union européenne et auxquels doivent répondre les acteurs politiques n'ont pas manqué de stimuler les réflexions.

Différents groupes de réflexion ont formulé des propositions pour réformer l'UE et, plus particulièrement, l'Union économique et monétaire (UEM). Parmi ces dernières, celles du Groupe allemand de Glienicke sont celles qui ont à l'heure actuelle eu le plus d'échos ${ }^{(23)}$. Ses membres ont même été invités à la fois par le président fédéral, Joachim Gauck, et par le président du Conseil européen, Herman van Rompuy, à venir leur présenter leurs propositions. En guise de réponse, du côté français, le Groupe Eiffel Europe a publié quelques mois plus tard un texte intitulé «Pour une Communauté politique de l'euro " ${ }^{(24)}$. Mais diverses autres propositions ont également été faites telles que le rapport réalisé par Pierre de Boissieu, Tom de Bruijn, Antonio Vitorino et Stephen Wall ${ }^{(25)}$ qui a été remis à François Hollande en octobre 2013, ou encore celles proposées par l'Institut Montaigne ${ }^{(26)}$, ou enfin le «Manifeste pour une Union politique de l'euro » signé entre autres par Guillaume Duval, Pierre Rosanvallon et Thomas Piketty.

Plusieurs éléments peuvent être dégagés de ces propositions, essentiellement d'origine française et allemande. En premier lieu, il existe une tendance très claire sur le fait que les réformes institutionnelles à venir vont essentiellement être consacrées à la zone euro. Il n'y a par contre pas de consensus sur la méthode et les mesures à prendre pour y parvenir. Pour certains, il convient de travailler directement sur un nouveau traité, pour d'autres, ceci sera certes nécessaire à terme mais pas dans l'immédiat, alors que d'autres encore estiment qu'il est possible de prendre les

23 Groupe de Glienicke, « Vers une Union de l'euro », octobre 2013, disponible sur: http://www.notreeurope.eu/media/tribune1lallemandsne-ijdoct2013.pdf?pdf=ok.

24 Groupe Eiffel Europe, "Pour une Communauté politique de l’Euro », disponible sur: http://www. groupe-eiffel.eu/pour-une-communaute-politique-de-leuro/.

25 Rapport « Refaire l'Europe: esquisse d'une politique» (note 2).

26 Jean-Paul Tran Thiet (dir.), "Comment renforcer l'Europe politique », Institut Montaigne, janvier 2014, disponible sur: http://www.institutmontaigne.org/fr/publications/comment-renforcerleurope-politique. 
mesures nécessaires à traités constants ${ }^{(27)}$. A contrario, on relève un large accord sur la nécessité d'un gouvernement économique européen, mais les précisions quant à sa composition, son mode de nomination ou les moyens pour le contrôler sont encore à déterminer. Il existe aussi une tendance forte sur la nécessité d'un budget propre pour la zone euro mais les opinions divergent quant à son montant et au moyen de l'alimenter (soit via des ressources propres, soit via un pourcentage des PIB nationaux). Ils mettent aussi l'accent sur la subsidiarité, mais pour certains elle doit aller de pair avec la possibilité de sanctionner les États. Enfin, il y a une insistance commune sur l'importance du contrôle démocratique assuré par une assemblée dédiée à la zone euro. Toutefois, la composition de cette assemblée reste à définir entre plusieurs options: des députés européens ou des députés nationaux désignés, ou bien des députés élus spécialement.

Compte tenu de la fracture qui existe aujourd'hui entre les citoyens et l'Europe, la légitimation démocratique des décisions prises au sein de la zone euro est un élément central. L'accroissement des pouvoirs du Parlement européen a été la réponse généralement apportée aux accusations de déficit démocratique formulées à l'encontre de l'UE. Mais malgré des responsabilités croissantes à chaque révision de traité, les taux de participation aux élections européennes n'ont cessé de chuter. Ils ont désormais atteint des niveaux tellement bas que la légitimité du Parlement européen elle-même en vient à être remise en question. Dans le même ordre d'idées, adopter à nouveau d'importantes modifications de la structure européenne sans avoir un consentement populaire pourrait constituer une fracture irréparable pour le projet européen. Si la voie référendaire apparaît comme vouée à l'échec, l'ouverture d'un vaste débat dont l'issue serait incertaine et qui pourrait conduire à certaines décisions radicales semble néanmoins essentielle.

Depuis le début des années 2000, la gravité de la situation a incité le philosophe Jürgen Habermas à concentrer son énergie sur la question européenne ${ }^{(28)}$. L'ambition constitutionnelle apparue à ce moment-là a alimenté les débats et suscité des espérances, vite déçues par l'échec manifeste du processus. Si l'enthousiasme fédéraliste de Jürgen Habermas apparaît en net décalage avec les constats dressés au début de cette contribution, il nourrit le débat sur l'interaction entre les États nations et les structures supranationales. Pour le philosophe suisse Francis Cheneval, la solution consiste précisément à combler les lacunes au niveau national. Ces dernières sont liées à des interactions insuffisantes entre les instances européennes et nationales. Il insiste avant tout sur une prise en compte et une implication plus forte des parlements nationaux mais également des juridictions nationales ${ }^{(29)}$. La volonté politique, en vogue depuis plusieurs années, de renforcer le rôle des parlements nationaux sur les affaires

27 Pour plus de précision à ce sujet et une contribution au débat, voir l'analyse de Thierry CHOPIN, «Réformer l'Union européenne: Quelles méthodes? Quels scénarios? ", Questions d'Europe, n³20, Fondation Robert Schuman, 7 juillet 2014, disponible en ligne: http://www.robert-schuman.eu/fr/ doc/questions-d-europe/qe-320-fr.pdf.

28 Voir à ce sujet notamment le dossier spécial "Habermas et l’Europe ", paru dans Allemagne d'aujourd'hui, n’207, janvier-mars 2014.

29 Interview de Francis Cheneval, "Skeptiker stärken EU-Demokratie ", Magazine de l'université de Zurich, p. 52-55, http://www.nccr-democracy.uzh.ch/media_articles/uzhmagazin20142.pdf. 
européennes s'inscrit précisément dans cette optique ${ }^{(30)}$. C’est sans doute dans ce chaînon manquant, entre la sphère européenne et les sphères nationales, que réside pour partie la réponse aux défis actuels.

\section{Conclusion}

Si une manne de textes est présente pour faire face à la situation et répondre aux besoins, il s'avère désormais nécessaire d'éviter la panne des réalisations. À l'instar de ce qu'avance le philosophe Jean-Marc Ferry, l'échec du projet européen ne risque pas de venir des ambitions et utopies, mais de l'" apathie politique » ${ }^{(31)}$. Pour une fois, les voies du compromis politique entre les États membres ne semblent pourtant pas être les plus complexes. Le large consensus existant sur la nécessité de concentrer les travaux sur l'Union économique et monétaire et d'améliorer la gouvernance de la zone euro offre un objectif clair. De même, une voie médiane est en mesure de se dégager pour une démarche en deux temps consistant à prendre un maximum de mesures possibles à traité constant tout en ouvrant la voie d'une refonte plus globale dans laquelle aussi bien la question de la place du Royaume-Uni que celle de l'articulation entre les sphères d'intégration différenciées seront traitées.

In fine, la difficulté résidera essentiellement dans le défi que représente la question démocratique. L'implication des États membres dans la sphère supranationale ainsi que la manière dont l'Allemagne parviendra à gérer sa position seront également des variables fondamentales, mais le lien entre le projet et ceux qui l'animent est la condition de sa persistance.

30 Voir à ce sujet l'étude «Democratic Control in the Member States of the European Council and the Eurozone summits ", réalisée pour le Parlement européen par Wolfgang Wessels, Olivier RozENBERG et al., janvier 2013, http://www.notre-europe.eu/media/democraticcontrol-rozenberg-kreilinger-etal-ne-jdi-tepsa-mar13.pdf?pdf=ok.

31 Jean-Marc Ferry, La République crépusculaire, Paris, Le Cerf, 2010, p. 60. 